\title{
Synergic hypocholesterolaemic effect of $n-3$ PUFA and oestrogen by modulation of hepatic cholesterol metabolism in female rats
}

\author{
Yuna Oh, Youri Jin and Yongsoon Park* \\ Department of Food and Nutrition, Hanyang University, Seoul 133-791, South Korea \\ (Submitted 18 June 2015 - Final revision received 4 August 2015 - Accepted 12 August 2015 - First published online 21 September 2015)
}

\begin{abstract}
$n-3$ PUFA such as EPA and DHA as well as oestrogen have been reported to decrease blood levels of cholesterol, but their underlying mechanism is unclear. The purpose of this study was to determine the effects of the combination of $n-3$ PUFA supplementation and oestrogen injection on hepatic cholesterol metabolism. Rats were fed a modified AIN-93G diet with 0,1 or $2 \% n-3$ PUFA (EPA + DHA) relative to the total energy intake for 12 weeks. Rats were surgically ovariectomised at week 8, and, after 1-week recovery, rats were injected with $17 \beta$-oestradiol3-benzoate $\left(\mathrm{E}_{2}\right)$ or maize oil for the last 3 weeks. Supplementation with $n$-3 PUFA and $\mathrm{E}_{2}$ injection significantly increased the ratio of the hepatic expression of phosphorylated AMP activated protein kinase (p-AMPK):AMP activated protein kinase (AMPK) and decreased sterol regulatory element-binding protein-2, 3-hydroxy-3-methylglutaryl coenzyme A reductase and proprotein convertase subtilisin/kexin type 9. Supplementation with $n$-3 PUFA increased hepatic expression of cholesterol $7 \alpha$-hydroxylase (CYP7A1), sterol 12 $\alpha$-hydroxylase (CYP8B1) and sterol 27-hydroxylase (CYP27A1); however, $\mathrm{E}_{2}$ injection decreased CYP7A1 and CYP8B1 but not CYP27A1. Additionally, $\mathrm{E}_{2}$ injection increased hepatic expression of oestrogen receptor- $\alpha$ and $\beta$. In conclusion, $n-3$ PUFA supplementation and $\mathrm{E}_{2}$ injection had synergic hypocholesterolaemic effects by down-regulating hepatic cholesterol synthesis ( $n-3$ PUFA and oestrogen) and up-regulating bile acid synthesis (n-3 PUFA) in ovariectomised rats.
\end{abstract}

Key words: Cholesterol metabolism: Oestrogen: $n$-3 PUFA: Ovariectomised rats: Proprotein convertase subtilisin/kexin type 9

Menopause is associated with unfavourable blood-cholesterol changes such as elevated total cholesterol (TC), TAG and LDLcholesterol and reduced HDL-cholesterol ${ }^{(1)}$. Dyslipidaemia, especially elevated blood LDL-cholesterol, is a major risk factor for $\mathrm{CVD}^{(1)}$. Post-menopausal women have increased levels of LDL-cholesterol and TC as compared with pre-menopausal women, all of these contribute to an atherogenic lipid profile $^{(1,2)}$. Post-menopausal women are, therefore, at increased risk for CVD.

Oestrogen injection has been shown to decrease blood levels of LDL-cholesterol by reducing hepatic cholesterol synthesis via down-regulation of sterol regulatory element-binding protein-2 (SREBP-2) and 3-hydroxy-3-methylglutaryl coenzyme A reductase $(H M G-C O A \text { reductase })^{(2-4)}$. As a new drug target for lowering LDL-cholesterol, proprotein convertase subtilisin/kexin type 9 (PCSK9) is also decreased by oestrogen, suggesting that oestrogen prevents the degradation of the LDL receptor ${ }^{(5,6)}$. However, oestrogen has also been suggested to induce cholestasis by inhibiting biliary cholesterol secretion and bile acid synthesis via down-regulating expression of cholesterol $7 \alpha$-hydroxylase $(C Y P 7 A 1)^{(7,8)}$.
Unlike oestrogen, $n$-3 PUFA, EPA (20:5n-3) and DHA (22 : $6 n-3)$ have been shown to decrease cholesterol by increasing hepatic bile acid synthesis by up-regulation of $C Y P 7 A 1$, sterol $12 \alpha$-hydroxylase (CYP8B1) and sterol 27-hydroxylase $(C Y P 27 A 1)^{(9,10)}$. In addition to increasing synthesis of bile acids, $n$-3 PUFA decrease blood levels of cholesterol by downregulating $S R E B P-2$ and $H M G-C O A$ reductase and, thus, decreasing hepatic cholesterol synthesis ${ }^{(11,12)}$. However, the effect of $n-3$ PUFA on hepatic expression of PCSK9 has not been studied.

Bravo et al. ${ }^{(13)}$ reported that supplementation with $n-3$ PUFA reduced plasma levels of LDL-cholesterol in male rats injected with oestrogen by increasing the number of LDL receptors, but hepatic cholesterol synthesis was not studied. Although the effect of $n$-3 PUFA on cholesterol concentration is not entirely clear, a recent meta-analysis reported an increase in LDL-cholesterol by $n-3$ PUFA ${ }^{(14)}$. The LDL-cholesterolincreasing effects of $n-3$ PUFA have been shown in patients with hypertriacylglycerolaemia ${ }^{(15)}$ and hamsters consuming high-fat diets ${ }^{(16)}$ but not in healthy volunteers ${ }^{(17)}$. Therefore, the purpose of the present study was to determine the effects of the

Abbreviations: AMPK, AMP activated protein kinase; CYP7A1, cholesterol $7 \alpha$-hydroxylase; CYP8B1, sterol 12 $\alpha$-hydroxylase; CYP27A1, sterol 27-hydroxylase; $\mathrm{E}_{2}, 17 \beta$-oestradiol-3-benzoate; ER- $\alpha$, oestrogen receptor- $\alpha$; ER- $\beta$, oestrogen receptor- $\beta$; HMG-CoA reductase, 3-hydroxy-3-methylglutaryl coenzyme A reductase; p-AMPK, phosphorylated AMP activated protein kinase; SREBP-2, sterol regulatory element-binding protein-2; TC, total cholesterol.

*Corresponding author: Y. Park, fax +82 2220 1856, email yongsoon@hanyang.ac.kr 
combination of $n-3$ PUFA supplementation and oestrogen injection on hepatic cholesterol synthesis and breakdown, including the effects on PCSK9 expression, in ovariectomised rats (OVX) fed a low-fat diet.

\section{Methods}

\section{Animals}

A total of forty-eight female Wistar rats aged 3 weeks (Jungang Lab Animal Inc.) were used for the present study. All experimental protocols were adhered to the institutional guidelines for the care and management of laboratory animals, and approved by the Institutional Animal Care and Use Committee of Hanyang University (registration no. HY-IACUC-12-076).

\section{Study design}

Rats were individually housed under conditions of constant temperature $\left(22(\operatorname{SEM} 1)^{\circ} \mathrm{C}\right)$ and humidity (47 (SEM 1) \%) and a $12 \mathrm{~h}$ light $-12 \mathrm{~h}$ dark cycle. Food pellets and fresh tap water were available ad libitum and food intake was measured daily. The body weight of the rats was determined weekly for 12 weeks.

After 1 week of acclimation, rats were randomly divided into three isoenergetic diet groups ( $n 16$ each) and fed a modified American Institute of Nutrition (AIN) 93G diet for 12 weeks. Soyabean oil in the AIN-93G diet was substituted with same amount of grape seed oil (CJ CheilJedang) and/or fish oil (Cenovis Health Company) in order to achieve an EPA + DHA content of 0,1 or $2 \%$ of the total energy. Grape seed oil contained very low levels of $\alpha$-linolenic acid (18 : $3 n-3)$, EPA and DHA. Per kg of diet, there was $42.94 \mathrm{~g}$ total fat (10\% of energy) and 0,1 or $2 \% n$-3 PUFA (EPA+DHA) equal to $0,8.09$ or $16 \cdot 21 \mathrm{~g}$ of fish oil, respectively. The fatty acid composition of the diets is shown in Table 1 .

At week 8, rats were surgically ovariectomised under anaesthesia using a combination of tiletamine/zolazepam

Table 1. Fatty acid composition of the diets

\begin{tabular}{lrrr}
\hline Fatty acids (\%) & $0 \% n 3$ & $1 \% n 3$ & $2 \% n 3$ \\
\hline $14: 0$ & 0.09 & 0.16 & 0.23 \\
$16: 0$ & $6 \cdot 87$ & 6.70 & 6.53 \\
$16: 1 n-7$ & $0 \cdot 11$ & 0.15 & 0.18 \\
$18: 0$ & 3.63 & 4.20 & 4.78 \\
$18: 1 n-9$ & 14.72 & 14.12 & 13.53 \\
$18: 2 n-6$ & $73 \cdot 15$ & 59.58 & 45.95 \\
$18: 3 n-3$ & 0.44 & 0.47 & 0.49 \\
$20: 4 n-6$ & 0.01 & 0.34 & 0.67 \\
$20: 5 n-3$ & 0.02 & $6 \cdot 17$ & 12.34 \\
$22: 4 n-6$ & 0.02 & 0.35 & 0.68 \\
$22: 5 n-6$ & 0.01 & 0.12 & 0.24 \\
$22: 5 n-3$ & 0.02 & 0.86 & 1.71 \\
$22: 6 n-3$ & 0.01 & 4.86 & 9.74 \\
SFA & 10.61 & 11.11 & 11.62 \\
MUFA & 15.08 & 14.82 & 14.56 \\
$n-3$ PUFA & 0.48 & 12.36 & 24.28 \\
$n-6$ PUFA & 73.24 & 60.97 & 48.65 \\
$n-3: n-6$ ratio & 0.01 & 0.20 & 0.50 \\
\hline
\end{tabular}

0,1 and $2 \% n 3, \% n-3$ PUFA relative to the total energy intake in the diet.
(Zoletil, $25 \mathrm{ml} / \mathrm{kg}$; Virbac S.A.) and xylazine (Rumpun, $10 \mathrm{ml} / \mathrm{kg}$; Bayer Korea). Starting 1 week after ovariectomisation, rats ( $n$ 8/group) were randomly assigned to groups injected with either $17 \beta$-oestradiol-3-benzoate $\left(\mathrm{E}_{2}\right)$ or the vehicle only (maize oil; Sigma-Aldrich) for the last 3 weeks of the study (week 10-12); the six groups were: $0 \% n-3$ PUFA diet with maize oil injection $(0 \% n 3), 0 \% n-3$ PUFA diet with $\mathrm{E}_{2}$ injection $(0 \%$ $\left.n 3+\mathrm{E}_{2}\right), 1 \% n-3$ PUFA diet with maize oil injection (1\%n3), $1 \%$ $n$-3 PUFA diet with $\mathrm{E}_{2}$ injection $\left(1 \% n 3+\mathrm{E}_{2}\right), 2 \% n-3$ PUFA diet with maize oil injection ( $2 \% n 3)$ and $2 \% n-3$ PUFA diet with $\mathrm{E}_{2}$ injection $\left(2 \% n 3+E_{2}\right)$. At 12 week of the experiment, rats were fasted overnight and euthanised with an intraperitoneal injection of Zoletil $(25 \mathrm{ml} / \mathrm{kg})$ and Rumpun $(10 \mathrm{ml} / \mathrm{kg})$. Blood was collected, and serum was obtained after centrifugation. Organs were collected, washed with saline solution and weighed. Serum and tissue samples were stored at $-80^{\circ} \mathrm{C}$ until analysis.

\section{Determination of serum and liver lipid}

Hepatic lipid was extracted according to the method of Folch et $a l .{ }^{(18)}$. Hepatic and serum concentrations of TC, TAG and serum HDL-cholesterol were measured using commercial kits (Asan Pharmaceutical) according to manufacturer's instructions. The concentration of LDL-cholesterol was calculated using the formula of Friedewald et al. ${ }^{(19)}$.

\section{GC analysis}

Serum fatty acid composition was measured as previously reported $^{(20)}$. Briefly, $12.5 \mu \mathrm{l}$ of serum was methylated with $500 \mu \mathrm{l}$ of boron trifluoride methanol-benzene (Sigma-Aldrich) for $45 \mathrm{~min}$ at $100^{\circ} \mathrm{C}$. Fatty acid methyl esters were extracted with $500 \mu \mathrm{l}$ of hexane and analysed by GC (Shimadzu 2010) using a SP2560 capillary column $(100 \mathrm{~m} \times 0.25 \mathrm{~mm}$ i.d., $0.2 \mu \mathrm{m}$ film thickness, Supelco). Fatty acids were identified by comparing retention times with standards (GLC-727; Nu-Check Prep). Every batch was analysed with a quality control sample, and the $\mathrm{CV}$ was $<5 \%$.

\section{Western blot analysis}

For analysis, extracts were prepared by homogenising the liver tissue in lysis buffer $(0.25 \mathrm{~m}$-sucrose, $20 \mathrm{~mm}-\mathrm{N}$-2-hydroxyethylpiperazine- $N$-2-ethane sulfonic acid, 2 mm-dithiothreitol, $1 \mathrm{~mm}$-phenylmethanesulfonylfluoride, $0.5 \mathrm{~mm}$-EDTA, $10 \mu \mathrm{g} / \mathrm{ml}$ aprotinin, $10 \mu \mathrm{g} / \mathrm{ml}$ leupeptin and $1 \mathrm{~mm}-\mathrm{Na}_{3} \mathrm{VO}_{4}, \mathrm{pH}$ 7.5) plus a phosphatase inhibitor cocktail tablet (Roche Life Science). After centrifugation at $20000 \boldsymbol{g}$ for $1 \mathrm{~h}$ at $4^{\circ} \mathrm{C}$ (Eppendorf 5417R; Eppendorf), the supernatant was collected as a cytosolic fraction. To obtain the nuclear fraction, the pellets were re-suspended in lysis buffer with $1 \%(\mathrm{v} / \mathrm{v})$ Triton X-100, and centrifuged at 20000 $\boldsymbol{g}$ for $30 \mathrm{~min}$ at $4^{\circ} \mathrm{C}$. The supernatant was used for analysis of nuclear proteins. For protein quantification, the Bradford method was applied using bovine serum albumin (Bio-Rad) as a standard. Individual lysates (30-80 $\mu \mathrm{g}$ of protein) were separated on an $8 \%$ SDS-PAGE and transferred to polyvinylidene fluoride membranes ( $0.45 \mu \mathrm{m}$, Merck Millipore, Billerica, MA, USA).

Antibodies used in this study included the following: AMP activated protein kinase (AMPK) and phosphorylated AMPK 
(p-AMPK) from Cell Signaling Technology; HMG-CoA reductase, CYP7A1 and CYP8B1 from Santa Cruz Biotechnology; and SREBP-2, PCSK9, CYP27A1, oestrogen receptor- $\alpha$ (ER- $\alpha$ ) and oestrogen receptor- $\beta$ (ER- $\beta$ ) from Abcam.

After blocking with $5 \%$ skimmed milk or $5 \%$ bovine serum albumin in tris-buffered saline with $0.1 \%$ tween for $1 \mathrm{~h}$ at room temperature, membranes were incubated overnight at $4{ }^{\circ} \mathrm{C}$ with the following primary antibodies: AMPK (1:500), p-AMPK (1:1000), HMG-CoA reductase (1:100), CYP7A1 (1:250), CYP8B1 (1:250), SREBP-2 (1:1000), PCSK9 (1:1000), CYP27A1 (1:1000), ER- $\alpha$ (1:500) and ER- $\beta$ (1:500). Secondary antibody incubation was performed using horseradish peroxidase-conjugated antimouse IgG (1:5000; Enzo Life Science), anti-goat (1:10 000; Santa Cruz Biotechnology) or anti-rabbit (1:10000; Cell Signaling Technology) for $1 \mathrm{~h}$ at room temperature. The immunoreactive proteins were developed using the enhanced chemiluminescence kit (GE Healthcare Life Sciences) and quantified using the Chemidoc MP Imaging System (Bio-Rad). The relative amounts of the proteins were calculated by normalising to the quantity of $\beta$-actin (1:1000; BD Transduction Laboratories).

\section{Statistical analyses}

All data are expressed as mean values with their standard errors. Statistical differences were calculated with a two-way ANOVA. All the statistical analyses were performed using SPSS for Windows, version 18.0 (SPSS Inc.). $P$ values $<0.05$ were considered statistically significant.

\section{Results}

\section{Food intake, body weight and organ weight}

Supplementation with $n-3$ PUFA had no significant effect on dietary intake, body weight or organ weight (Table 2; see online Supplementary Table S1). $\mathrm{E}_{2}$ injection, however, significantly decreased dietary intake, final body weight and visceral fat weight, whereas it increased liver, uterus and kidney weight regardless of $n$ - 3 PUFA supplementation. $\mathrm{E}_{2}$ injection had no significant effect on the weight of the liver or kidneys.

\section{Serum and hepatic lipid}

Supplementation with n-3 PUFA significantly and dosedependently decreased the serum levels of TC, LDL-cholesterol and TAG, and the hepatic levels of TC and TAG (Table 3; see online Supplementary Table S2). Similarly, $\mathrm{E}_{2}$ injection significantly decreased the serum levels of LDL-cholesterol and TAG and the hepatic levels of TC and TAG. However, serum levels of $\mathrm{TC}$ were not significantly affected by $\mathrm{E}_{2}$ injection. Serum levels of HDL-cholesterol were significantly increased by $\mathrm{E}_{2}$ injection but not by $n$-3 PUFA supplementation. There was a significant synergistic effect of $n-3$ PUFA supplementation and $\mathrm{E}_{2}$ injection on serum LDL-cholesterol and hepatic TC levels.

\section{Serum fatty acid composition}

Supplementation with $n$-3 PUFA significantly increased serum levels of total $n-3$ PUFA, $20: 5 n-3,22: 5 n-3$ and $22: 6 n-3$, while

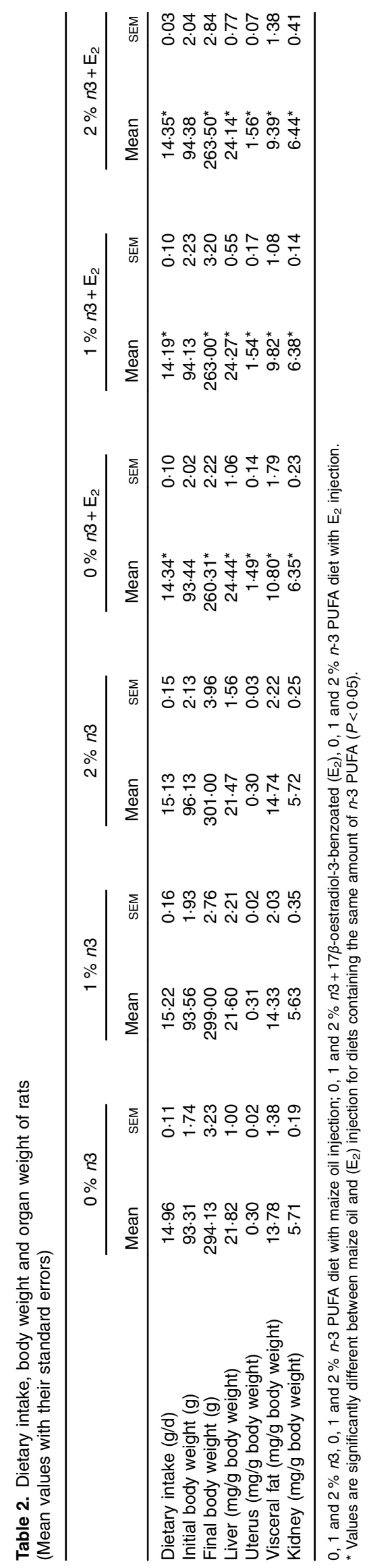


decreasing the serum levels of total $n-6$ PUFA $20: 4 n-6$ in a dose-dependent manner (see online Supplementary Table S3). $\mathrm{E}_{2}$ injection decreased the serum levels of $14: 0,16: 0,16: 1 n-7$ and $18: 1 n-9$ but increased the concentration of $18: 0$. In addition, $\mathrm{E}_{2}$ injection significantly increased the serum levels of $22: 5 n-3$ and $22: 6 n-3$ in rats fed $n-3$ PUFA. There was a significant interaction between $n$-3 PUFA supplementation and $\mathrm{E}_{2}$ injection on the serum levels of $22: 6 n-3$, total $n-3$ PUFA, 22 : 5n-6 and total n-6 PUFA.

\section{Hepatic protein expression related with cholesterol metabolism}

Supplementation with $n-3$ PUFA and $\mathrm{E}_{2}$ injection significantly decreased the expression of HMG-COA reductase, SREBP-2 and PCSK9, whereas it increased the ratio of the expression of p-AMPK:AMPK (Fig. 1). Hepatic expressions of p-AMPK and AMPK were also increased both by $n$-3 PUFA supplementation and $\mathrm{E}_{2}$ injection (see online Supplementary Fig. S1).

Supplementation with $n-3$ PUFA significantly increased the expression of CYP7A1, CYP8B1 and CYP27A1 (Fig. 2). However, $\mathrm{E}_{2}$ injection significantly decreased the expression of CYP8B1 but not of CYP27A1 (Fig. 2). In addition, $\mathrm{E}_{2}$ injection significantly increased the hepatic expression of ER- $\alpha$ and ER- $\beta$, but $n$-3 PUFA did not (Fig. 3).

\section{Discussion}

This was the first report to suggest that $\mathrm{E}_{2}$ injection and $n-3$ PUFA supplementation have a synergic hypocholesterolaemic effect through inhibited hepatic cholesterol synthesis and increased breakdown of hepatic cholesterol in OVX. Consistent with our results, Bravo et al. ${ }^{(13)}$ reported that $\mathrm{E}_{2}$ injection and $n$-3 PUFA supplementation decreased LDL-cholesterol by increasing the amount of LDL receptors in male rats. The LDL receptor binds with $P C S K 9$, which enhances LDL receptor degradation and results in increased plasma levels of LDLcholesterol $^{(21,22)}$. The present study showed that $\mathrm{E}_{2}$ injection and $n$-3 PUFA supplementation synergistically reduced hepatic expression of PCSK9, which inhibited LDL receptor degradation and decreased LDL-cholesterol. Previously, $\mathrm{E}_{2}$ injection decreased PCSK9 expression in male rats ${ }^{(5)}$, but the effect of n-3 PUFA had not been investigated.

PCSK9 has been shown to be regulated by SREBP-2, which communicates with hepatic proteins involved in cholesterol synthesis such as p-AMPK, AMPK and HMG-CoA reductase ${ }^{(23)}$. Previous preclinical studies have suggested that $\mathrm{E}_{2}$ injection ${ }^{(4,24)}$ or $n$-3 PUFA supplementation ${ }^{(11,25)}$ reduce cholesterol synthesis by increasing $A M P K$ phosphorylation, reducing expression of $H M G-C O A$ reductase and reducing expression of SREBP-2. Consistently, the present study shows that the reduced hepatic cholesterol synthesis was due to an up-regulation of p-AMPK and AMPK, an increase in the P-AMPK:AMPK ratio, and a downregulation of HMG-COA reductase and SREBP-2. However, reports on the effects of $n-3$ PUFA on LDL-cholesterol have been contradictory; some have proposed that rapid TAG clearance by lipoprotein lipase can promote the conversion of VLDL to LDL and thus increase circulating LDL-cholesterol ${ }^{(26)}$. 
(a)

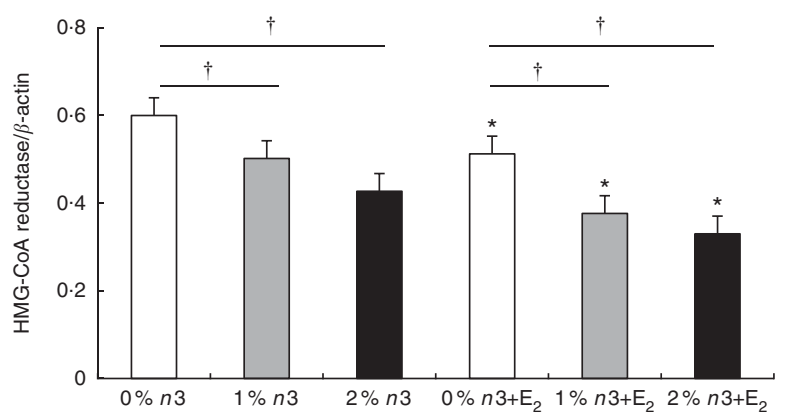

(c)

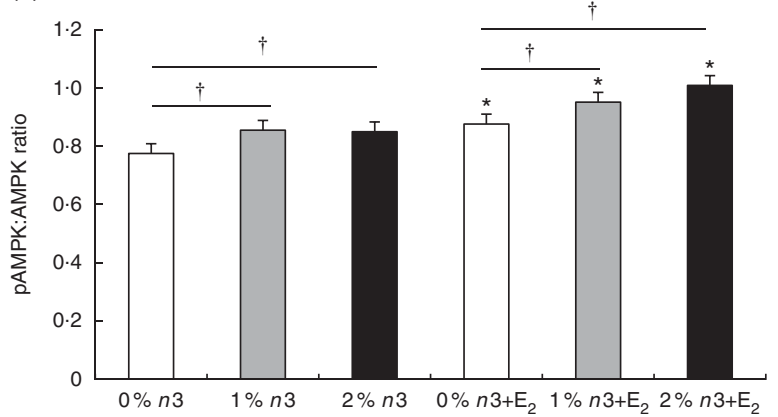

(b)

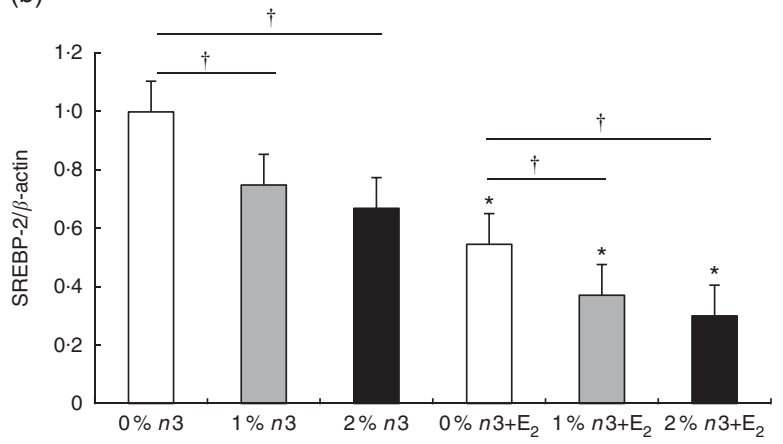

(d)

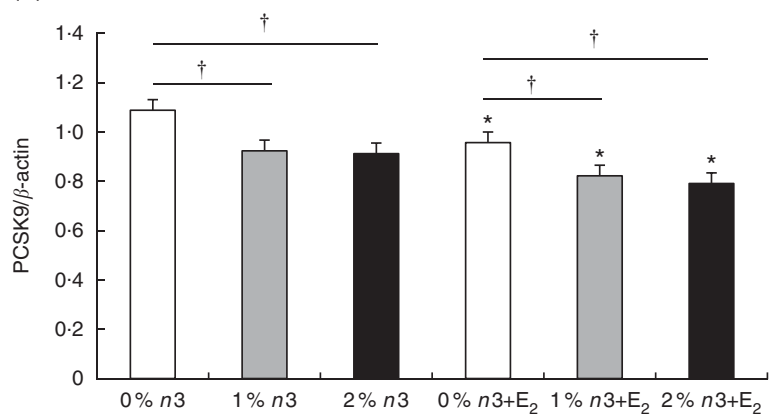

Fig. 1. The effect of $n-3$ PUFA supplementation and 17 $\beta$-oestradiol-3-benzoated $\left(\mathrm{E}_{2}\right)$ injection on expression of (a) 3-hydroxy-3-methylglutaryl coenzyme $A$ reductase (HMG-CoA reductase), (b) sterol regulatory element-binding protein-2 (SREBP-2), (c) phosphorylated AMP activated protein kinase ( $\mathrm{p}$-AMPK):AMP activated protein kinase (AMPK) ratio and (d) proprotein convertase subtilisin/kexin type 9 (PCSK9) in the liver of ovariectomised rats. Values are means ( $n 8$ rats/group), with their standard errors represented by vertical bars. * Mean values are significantly different between maize oil and $E_{2}$ injection for diets containing the same amount of $n$-3 PUFA $(P<0.05)$. † Mean values are significantly different among 0,1 and $2 \% n 3$ within the maize oil and $E_{2}$ injected groups. 0,1 and $2 \% n 3,0,1$ and $2 \% n-3$ PUFA diets with maize oil injection; 0,1 and $2 \% n 3+E_{2}, 0,1$ and $2 \% n-3$ PUFA diets with $E_{2}$ injection $(P<0.05)$.

The LDL-cholesterol-increasing effects of $n-3$ PUFA have been reported in hypertriacylglycerolaemia ${ }^{(15)}$, but not in normotriacylglycerolaemia ${ }^{(17)}$, as in the present study.

Another mechanism that has been shown to reduce blood levels of cholesterol is increased hepatic cholesterol breakdown. Bile acids promote hepatic cholesterol removal, and thus an increase in bile acid synthesis results in a reduction of blood cholesterol $^{(10)}$. Previous studies reported that $n-3$ PUFA supplementation increased hepatic expression of $C Y P 7 A 1, C Y P 8 B 1$ and $C Y P 27 A 1^{(9,10)}$ in mice, suggesting enhanced bile acid synthesis and reduced hepatic levels of cholesterol. On the other hand, oestrogen has been shown to decrease hepatic synthesis of bile acids through a reduction of CYP7A1 and CYP8B1 but not CYP27A1, in rats and mice ${ }^{(8,27)}$. Similarly, the present study showed that $E_{2}$ injection suppressed hepatic expression of CYP7A1 and CYP8B1 but not CYP27A1. The effect of oestrogen could be related to the expression of endoplasmic reticulumlocalised enzymes such as $C Y P 7 A 1$ but not the mitochondrialocalised enzyme $C Y P 27 A 1^{(28)}$. CYP7A1 regulates $C Y P 8 B 1$, which mediates bile acid synthesis through cholic acid synthesis ${ }^{(29)}$, and the suppression of $C Y P 8 B 1$ (e.g. by $\mathrm{E}_{2}$ ) causes a reduction of CYP7A1 expression. Although $\mathrm{E}_{2}$ injection did not increase the hepatic synthesis of bile acids in this study, it suppressed hepatic cholesterol synthesis that, in turn, decreased hepatic and blood levels of cholesterol. Previous studies have suggested that oestrogen induces cholestasis through reducing hepatic CYP7A1 and biliary cholesterol secretion ${ }^{(8,27)}$. Therefore, it is worth noting that the combination of $n-3$ PUFA and $\mathrm{E}_{2}$ in the present study could have decreased blood and hepatic levels of cholesterol by decreasing hepatic cholesterol synthesis and increasing hepatic synthesis of bile acids.

In the liver, $\mathrm{E}_{2}$ binds to oestrogen receptors (ER), specifically ER- $\alpha$ and ER- $\beta$ subtypes, to modulate cholesterol metabolism. The binding of $E_{2}$ to $E R$ results in the recruitment of co-activators and the displacement of co-repressors at DNA binding sites and thereby the modulation of gene and protein expression $^{(30)}$ including LDL receptors in liver. Thus, oestrogen can up-regulate the expression of the LDL receptor through binding to ER and increasing the clearance of blood LDL-cholesterol $^{(31)}$. In the present study, $\mathrm{E}_{2}$ injection increased the hepatic expression of $E R-\alpha$ and $E R-\beta$, but $n-3$ PUFA did not. This result is consistent with a previous study in which oestrogen increased the amount of hepatic ER in OVX rats ${ }^{(32)}$. However, the effect of $n$-3 PUFA on ER has not yet been reported.

In the present study, $\mathrm{E}_{2}$ injection decreased serum levels of $14: 0,16: 0,16: 1 n-7$ and $18: 1 n-9$ but increased $18: 0$. The elongase 6 enzyme elongates $16: 0$ to 18 : 0 ; this was demonstrated in elongase 6 knockout mice who exhibited greater concentrations of 16 : 0 but less 18 : 0 compared with wild-type mice ${ }^{(33)}$. In addition, elongase 6 expression was higher in females than males and was increased in oestrogentreated HepG2 cells $^{(34)}$. Consistently, the present study showed 
(a)

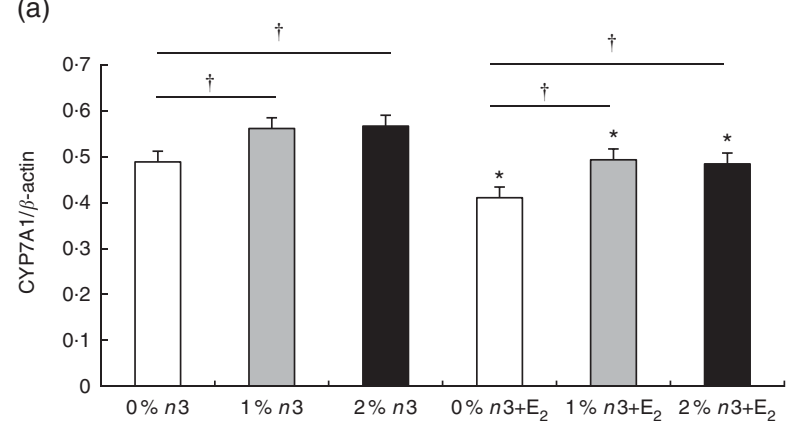

(b)

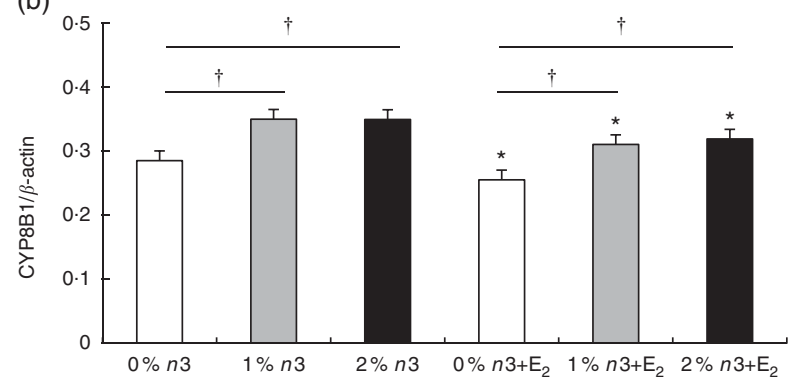

(c)

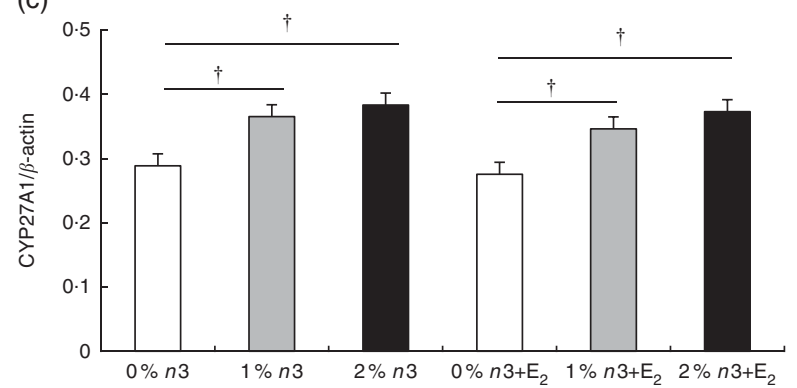

Fig. 2. The effect of $n-3$ PUFA supplementation and 17 $\beta$-oestradiol-3benzoated $\left(E_{2}\right)$ injection on the expression of (a) cholesterol $7 a$-hydroxylase (CYP7A1), (b) sterol 12 -hydroxylase (CYP8B1) and (c) sterol 27-hydroxylase (CYP27A1) in the liver of ovariectomised rats. Values are means ( $n 8$ rats/ group), with their standard errors represented by vertical bars. * Mean values are significantly different between maize oil and $E_{2}$ injection for diets containing the same amount of $n-3$ PUFA $(P<0.05)$. $\dagger$ Mean values are significantly different among 0,1 and $2 \% n 3$ within the maize oil and $E_{2}$ injected groups. 0,1 and $2 \% n 3,0,1$ and $2 \% n-3$ PUFA diets with maize oil injection; 0,1 and $2 \% n 3+E_{2}, 0,1$ and $2 \% n-3$ PUFA diets with $E_{2}$ injection $(P<0.05)$. that $\mathrm{E}_{2}$ injection increased $18: 0$ concentrations through activation of elongase 6. Additionally, oestrogen has been shown to inhibit stearoyl-CoA desaturase- ${ }^{(35)}$, the rate-limiting enzyme in the synthesis of MUFA. In the present study, concentrations of MUFA $16: 1 n-7$ and $18: 1 n-9$ were depressed in rats injected with $\mathrm{E}_{2}$. Furthermore, $\mathrm{E}_{2}$ injection and $n-3$ PUFA supplementation increased serum levels of $22: 5 n-3$ and $22: 6 n-3$ synergistically. $\mathrm{E}_{2}$ injection increased serum levels of $22: 5 n-6$ in the $0 \% n 3$ diet, as observed previously in plasma in rats ${ }^{(36)}$. Previous studies reported that dietary $n$ - 3 PUFA $^{(37,38)}$ and oestrogen $^{(36)}$ increased blood levels of $n-3$ PUFA and decreased levels of $n$-6 PUFA. Oestrogen increases $\Delta 6$-desaturase, which converts $18: 3 n-3$ to $20: 5 n-3$ and $22: 6 n-3^{(36)}$.

This study had a few limitations. Although rats are the most commonly used model for lipoprotein research, lipoprotein metabolism differs between rats and human. Differences are the efficient mechanisms for clearance of remnant-removal pathways and absence of cholesteryl ester transfer reaction ${ }^{(39)}$. There was no sham surgery group in the present study, but previous studies compared cholesterol metabolism between OVX and sham rats ${ }^{(3,40)}$. In addition, the present study did not measure bile acid concentrations of faecal or biliary secretion.

In conclusion, $n-3$ PUFA supplementation and $\mathrm{E}_{2}$ injection significantly reduced hepatic and blood levels of cholesterol by inhibiting hepatic cholesterol synthesis through increasing AMPK phosphorylation, decreasing PCSK9, SREBP-2 and HMGCoA reductase expression, and enhancing hepatic cholesterol breakdown through CYP7A1, CYP8B1 and CYP27A1. Further research is needed to determine the effects of $n-3$ PUFA supplementation and hormone replacement therapy on cholesterol metabolism in post-menopausal women.

\section{Acknowledgements}

The present study was supported by a Korean Research Foundation grant funded by the Korean Government (grant no. NRF-2012R1A1A2040553).

The authors' contributions are as follows: Y. O. carried out the experimental work and prepared the manuscript; Y. J. conducted laboratory and statistical analysis; Y. P. edited (a)

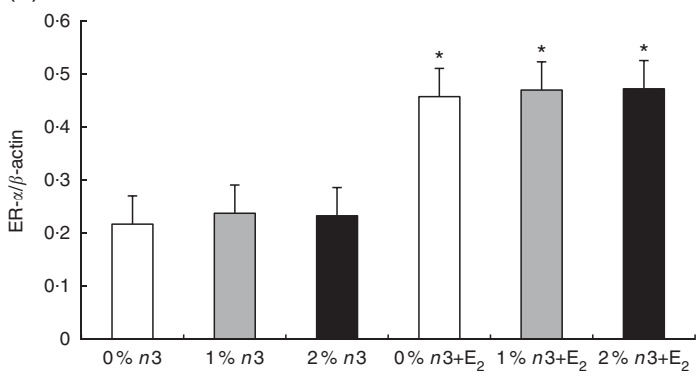

(b)

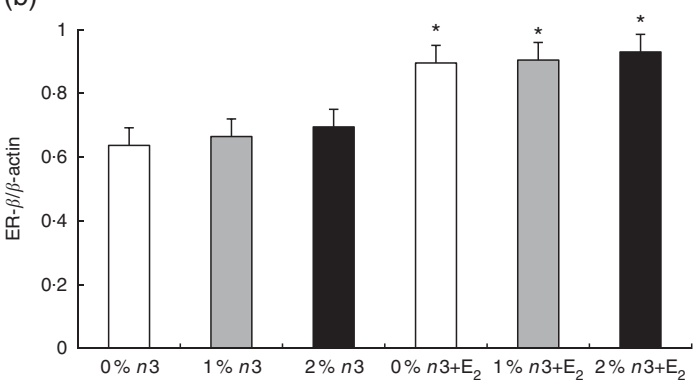

Fig. 3. The effect of $n$-3 PUFA supplementation and $17 \beta$-oestradiol-3-benzoated $\left(E_{2}\right)$ injection on expression of (a) oestrogen receptor- $a$ (ER- $a$ ) and (b) oestrogen receptor- $\beta(E R-\beta)$ in the liver of ovariectomised rats. Values are means ( $n 8$ rats/group), with their standard errors represented by vertical bars. ${ }^{\star}$ Mean values are significantly different between maize oil and $\mathrm{E}_{2}$ injection for diets containing the same amount of $n-3$ PUFA $(P<0.05) .0,1$ and $2 \% n 3,0,1$ and $2 \% n$-3 PUFA diets with maize oil injection; 0,1 and $2 \% n 3+E_{2}, 0,1$ and $2 \% n-3$ PUFA diets with $\mathrm{E}_{2}$ injection $(P<0.05)$. 
manuscript and has primary responsibility for the final content. All authors read and approved the final manuscript.

None of the authors has any conflicts of interest to declare.

\section{Supplementary material}

To view supplementary material for this article, please visit http://dx.doi.org/10.1017/S0007114515003517

\section{References}

1. Phan BA \& Toth PP (2014) Dyslipidemia in women: etiology and management. Int J Womens Health 6, 185-194.

2. Moorthy K, Yadav UC, Mantha AK, et al. (2004) Estradiol and progesterone treatments change the lipid profile in naturally menopausal rats from different age groups. Biogerontology $\mathbf{5}$, 411-419.

3. Nigro M, Santos AT, Barthem CS, et al. (2014) A change in liver metabolism but not in brown adipose tissue thermogenesis is an early event in ovariectomy-induced obesity in rats. Endocrinology 155, 2881-2891.

4. De Marinis E, Martini C, Trentalance A, et al. (2008) Sex differences in hepatic regulation of cholesterol homeostasis. $J$ Endocrinol 198, 635-643.

5. Persson L, Galman C, Angelin B, et al. (2009) Importance of proprotein convertase subtilisin/kexin type 9 in the hormonal and dietary regulation of rat liver low-density lipoprotein receptors. Endocrinology 150, 1140-1146.

6. Ghosh M, Galman C, Rudling M, et al. (2015) Influence of physiological changes in endogenous estrogen on circulating PCSK9 and LDL cholesterol. J Lipid Res 56, 463-469.

7. Davis RA, Elliott TS, Lattier GR, et al. (1986) Regulation of bile acid synthesis via direct effects on the microsomal membrane. Biochemistry 25, 1632-1636.

8. Cuevas MJ, Mauriz JL, Almar M, et al. (2001) Effect of epomediol on ethinyloestradiol-induced changes in bile acid and cholesterol metabolism in rats. Clin Exp Pharmacol Physiol 28, 637-642.

9. Berard AM, Dumon MF \& Darmon M (2004) Dietary fish oil up-regulates cholesterol 7alpha-hydroxylase mRNA in mouse liver leading to an increase in bile acid and cholesterol excretion. FEBS Lett 559, 125-128.

10. Kamisako T, Tanaka Y, Ikeda T, et al. (2012) Dietary fish oil regulates gene expression of cholesterol and bile acid transporters in mice. Hepatol Res 42, 321-326.

11. Boschetti E, Di Nunzio M, Danesi F, et al. (2013) Influence of genotype on the modulation of gene and protein expression by $n$-3 LC-PUFA in rats. Genes Nutr 8, 589-600.

12. Ramaprasad TR, Srinivasan K, Baskaran V, et al. (2006) Spray-dried milk supplemented with alpha-linolenic acid or eicosapentaenoic acid and docosahexaenoic acid decreases HMG Co A reductase activity and increases biliary secretion of lipids in rats. Steroids 71, 409-415.

13. Bravo E, Cantafora A, Cicchini C, et al. (1999) The influence of estrogen on hepatic cholesterol metabolism and biliary lipid secretion in rats fed fish oil. Biochim Biophys Acta 1437, 367-377.

14. Wei MY \& Jacobson TA (2011) Effects of eicosapentaenoic acid versus docosahexaenoic acid on serum lipids: a systematic review and meta-analysis. Curr Atheroscler Rep 13, 474-483.

15. Mori TA, Burke V, Puddey IB, et al. (2000) Purified eicosapentaenoic and docosahexaenoic acids have differential effects on serum lipids and lipoproteins, LDL particle size, glucose, and insulin in mildly hyperlipidemic men. Am J Clin Nutr 71, 1085-1094.
16. Ishida T, Ohta M, Nakakuki M, et al. (2013) Distinct regulation of plasma LDL cholesterol by eicosapentaenoic acid and docosahexaenoic acid in high fat diet-fed hamsters: participation of cholesterol ester transfer protein and LDL receptor. Prostaglandins Leukot Essent Fatty Acids 88, 281-288.

17. Grimsgaard S, Bonaa KH, Hansen JB, et al. (1997) Highly purified eicosapentaenoic acid and docosahexaenoic acid in humans have similar triacylglycerol-lowering effects but divergent effects on serum fatty acids. Am J Clin Nutr $\mathbf{6 6}$, 649-659.

18. Folch J, Lees M \& Sloane Stanley GH (1957) A simple method for the isolation and purification of total lipides from animal tissues. J Biol Chem 226, 497-509.

19. Friedewald WT, Levy RI \& Fredrickson DS (1972) Estimation of the concentration of low-density lipoprotein cholesterol in plasma, without use of the preparative ultracentrifuge. Clin Chem 18, 499-502.

20. Harris WS, von Schacky C \& Park Y (2013) Standardizing methods for assessing omega-3 fatty acid biostatus. In The Omega-3 Fatty Acid Deficiency Syndrome: Opportunities for Disease Prevention, pp. 385-398 [RK McNamara, editor]. Hauppauge, NY: Nova Science Publishers.

21. Zaid A, Roubtsova A, Essalmani R, et al. (2008) Proprotein convertase subtilisin/kexin type 9 (PCSK9): hepatocyte-specific low-density lipoprotein receptor degradation and critical role in mouse liver regeneration. Hepatology 48, 646-654.

22. Rashid S, Curtis DE, Garuti R, et al. (2005) Decreased plasma cholesterol and hypersensitivity to statins in mice lacking PCSK9. Proc Natl Acad Sci U S A 102, 5374-5379.

23. Horton JD, Cohen JC \& Hobbs HH (2007) Molecular biology of PCSK9: its role in LDL metabolism. Trends Biochem Sci $\mathbf{3 2}$, $71-77$.

24. Trapani L, Violo F \& Pallottini V (2010) Hypercholesterolemia and 3-hydroxy-3-methylglutaryl coenzyme A reductase regulation in aged female rats. Exp Gerontol 45, 119-128.

25. Suchankova G, Tekle M, Saha AK, et al. (2005) Dietary polyunsaturated fatty acids enhance hepatic AMP-activated protein kinase activity in rats. Biochem Biophys Res Commun 326, 851-858.

26. Park Y \& Harris WS (2003) Omega-3 fatty acid supplementation accelerates chylomicron triglyceride clearance. J Lipid Res 44, 455-463.

27. Yamamoto Y, Moore R, Hess HA, et al. (2006) Estrogen receptor alpha mediates 17alpha-ethynylestradiol causing hepatotoxicity. J Biol Chem 281, 16625-16631.

28. Koopen NR, Post SM, Wolters H, et al. (1999) Differential effects of 17alpha-ethinylestradiol on the neutral and acidic pathways of bile salt synthesis in the rat. J Lipid Res $\mathbf{4 0}$, 100-108.

29. Chiang JY (2004) Regulation of bile acid synthesis: pathways, nuclear receptors, and mechanisms. J Hepatol 40, 539-551.

30. Moolman JA (2006) Unravelling the cardioprotective mechanism of action of estrogens. Cardiovasc Res 69, 777-780.

31. Parini P, Angelin B \& Rudling M (1997) Importance of estrogen receptors in hepatic LDL receptor regulation. Arterioscler Thromb Vasc Biol 17, 1800-1805.

32. Sahlin L, Elger W, Hedden A, et al. (2002) Effects of estradiol and estradiol sulfamate on the liver of ovariectomized or ovariectomized and hypophysectomized rats. J Steroid Biochem Mol Biol 80, 457-467.

33. Matsuzaka T, Shimano H, Yahagi N, et al. (2007) Crucial role of a long-chain fatty acid elongase, Elovl6, in obesity-induced insulin resistance. Nat Med 13, 1193-1202.

34. Marks KA, Kitson AP \& Stark KD (2013) Hepatic and plasma sex differences in saturated and monounsaturated fatty acids 
are associated with differences in expression of elongase 6, but not stearoyl-CoA desaturase in Sprague-Dawley rats. Genes Nutr 8, 317-327.

35. Paquette A, Wang D, Jankowski M, et al. (2008) Effects of ovariectomy on PPAR alpha, SREBP-1c, and SCD-1 gene expression in the rat liver. Menopause 15, 1169-1175.

36. Kitson AP, Marks KA, Shaw B, et al. (2013) Treatment of ovariectomized rats with 17beta-estradiol increases hepatic delta- 6 desaturase enzyme expression and docosahexaenoic acid levels in hepatic and plasma phospholipids. Prostaglandins Leukot Essent Fatty Acids 89, 81-88.

37. Nakanishi A, Iitsuka N \& Tsukamoto I (2013) Fish oil suppresses bone resorption by inhibiting osteoclastogenesis through decreased expression of M-CSF, PU.1, MITF and RANK in ovariectomized rats. Mol Med Rep 7, 1896-1903.

38. Mohamed AI, Hussein AS, Bhathena SJ, et al. (2002) The effect of dietary menhaden, olive, and coconut oil fed with three levels of vitamin $\mathrm{E}$ on plasma and liver lipids and plasma fatty acid composition in rats. J Nutr Biochem 13, 435-441.

39. Oschry Y \& Eisenberg S (1982) Rat plasma lipoprotein: re-evaluation of a lipoprotein system in an animal devoid of cholesteryl ester transfer activity. J Lipid Res 23, 1099-1106.

40. van Lenten BJ, Melchior GW \& Roheim PS (1983) Lipoprotein metabolism in the ovariectomized rat. J Lipid Res $\mathbf{2 4}$ $1475-1484$. 